A note on algebraic extensions modulo I

Basma Gasmi and Noômen Jarboui 


\title{
ON ALGEBRAIC EXTENSIONS MODULO I
}

\author{
BASMA GASMI AND NOÔMEN JARBOUI
}

Received 06 October, 2014

\begin{abstract}
Let $I$ be a nonzero ideal of a ring $T$, let $\varphi: T \rightarrow E:=T / I$ denote the canonical projection, let $D$ be a ring contained in $E$, and let $R=\varphi^{-1}(D)$. The main purpose of this paper is to characterize when the ring extension $R \subset T$ is $n$ - (resp., universally) algebraic modulo $I$ in case $I$ is an intersection of finitely many maximal ideals of $T$.
\end{abstract}

2010 Mathematics Subject Classification: 13A15; 13B25; $13 \mathrm{C} 15$

Keywords: integral domain, prime ideal, algebraic extension, algebraic extension modulo $I$, residually algebraic extension, pullback

\section{INTRODUCTION}

All rings considered below are commutative with identity but not necessarily integral domains. All subrings and inclusions of rings are (unital) ring extensions; all ring/algebra homomorphisms are unital. Let $A$ be a ring and $n \geq 1$ be an integer. We denote by $A[n]$ the ring of polynomials in $n$ indeterminates over $A$ (for $n=1$, $A[1]=A[X]$ is the ring of polynomials in one indeterminate). For convenience, we write $A=A[0]$.

Let $I$ be a nonzero ideal of a $\operatorname{ring} T, \varphi: T \rightarrow E:=T / I$ the natural projection, and $D$ a ring contained in $E$. Then $R=\varphi^{-1}(D)$ is the ring arising from the following pullback of canonical homomorphisms:

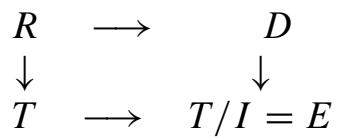

Following [4], we say that $R$ is the ring of the $(T, I, D)$ construction and we set $R:=(T, I, D)$. We shall assume that $D$ is properly contained in $E$ (and hence, that $R$ is properly contained in $T$ ), and we shall refer to this as a pullback diagram of type ( $\square$ ). If $I$ is an intersection of finitely many maximal ideals of $T$, we shall refer to this as a diagram $\left(\square_{\cap}\right)$. A very good account of pullback constructions has been given in [4,5] and [6]. It has fashionable in recent years to study rings via pullback diagrams. It is well worth noting that pullback constructions provide a rich source of examples and counterexamples in commutative algebra (see [1-5, 11, 12]). Unless 
otherwise specified, the symbols $T, D, I, R$ have the above meaning throughout the paper.

In [8] the authors introduced the concept of $n$-algebraic extension modulo $I$ for a diagram ( $\square$ ) when $T$ and $D$ are integral domains and $n \geq 0$ is an integer. More precisely, the ring extension $R \subset T$ (of integral domains) is said to be $n$-algebraic modulo $I$ if for every two prime ideals $Q^{\prime} \subset Q$ of $T[n]$ such that $I[n] \nsubseteq Q^{\prime}, I[n] \subseteq Q$ and $h t\left(Q \cap R[n] / Q^{\prime} \cap R[n]\right)=1$, then $R[n] /(Q \cap R[n]) \subseteq T[n] / Q$ is algebraic. This concept was first used to characterize when an integral domain $R$ of the form $D+I$, (where $I$ is a nonzero ideal of an integral domain $T$ and $D$ is a subring of $T$ satisfying $D \cap I=(0))$ is a (stably) strong S-domain (cf. [8, Théorème 1.7]). In [2], the authors dealt with a more general situation and used this concept to characterize when a ring $R$ arising from a diagram ( $\square$ ) is a (stably) strong S-domain. The main purpose of this paper is to study $n$-algebraic extensions modulo $I$ for a diagram $\left(\square_{\cap}\right)$ in order to deepen our knowledge about such extensions. We first extend this notion to arbitrary commutative rings. Our motivation is an example constructed by Fontana et al (see [8, Exemple 1.8]) of a diagram $\left(\square_{\cap}\right)$ in order to produce a ring extension $R \subset T$ which is 0 -algebraic modulo $I$ but not 1 -algebraic modulo $I$. For this reason, M. Fontana et al (see [8]) have introduced the following definition: The ring extension $R \subset T$ is said to be universally algebraic modulo $I$, if $R \subset T$ is $n$-algebraic modulo $I$ for each positive integer $n$. Our contribution (see Theorem 1) is to prove that for a diagram ( $\left.\square_{\cap}\right), R \subset T$ is $n$-algebraic modulo $I$ if and only if $R \subset T$ is 1-algebraic modulo $I$ if and only if $R \subset T$ is a residually algebraic extension. The key step (Lemma 1) is to show, for any diagram ( $\square$ ), that if $R \subset T$ is $n$-algebraic modulo $I$ (where $n \geq 1$ ), then $R \subset T$ is $(n-1)$-algebraic modulo $I$.

Throughout the paper, we use " $\subset$ " to denote proper containment and " $\subseteq$ " to denote containment. Transcendence degrees paly an important role in our study; if $A \subseteq B$ are two domains, we denote by $\operatorname{tr} \cdot \operatorname{deg}[B: A]$ the transcendence degree of the quotient field of $B$ over that of $A$. Any unexplained terminology is standard as in $[9,10]$. Relevant terminology and results will be recalled as needed through the paper.

\section{MAIN RESULTS}

We extend Fontana-Izelgue-Kabbaj's definition, mentioned in the introduction, to arbitrary commutative rings in the following way:

Definition 1. Let $n \geq 0$ be an integer. For a diagram ( $\square$ ), the extension $R \subset T$ is said to be $n$-algebraic modulo $I$ if for every two prime ideals $Q^{\prime} \subset Q$ of $T[n]$ such that $I[n] \not \subseteq Q^{\prime}, I[n] \subseteq Q$ and $h t\left(Q \cap R[n] / Q^{\prime} \cap R[n]\right)=1$, then $R[n] /(Q \cap R[n]) \subseteq$ $T[n] / Q$ is algebraic.

Definition 2. For a diagram ( $\square$ ), the extension $R \subset T$ is said to be universally algebraic modulo $I$ if $R \subset T$ is $n$-algebraic modulo $I$ for each integer $n \geq 0$. 
Recall that an extension of rings $A \subseteq B$ is said to be residually algebraic if for each prime ideal $Q$ of $B$, the extension $A /(Q \cap A) \subseteq B / Q$ is algebraic. It is clear that if $R \subset T$ is a residually algebraic extension, then so is $R[n] \subset T[n]$ for any positive integer $n$ (cf. [7, Lemme 1.4]). Hence $R \subset T$ is universally algebraic modulo $I$.

Recall from [10, Section 1-5] that if $p$ is a prime ideal of a ring $A$, and $Q$ is a prime ideal of $A[X]$ with $Q \cap A=p$, but with $Q \neq p[X]$, then we call $Q$ an upper to $p$ in $A[X]$ (or more simply, an upper to $p$, or just an upper).

The main result of this paper is the following theorem which identifies $n$-algebraic extensions modulo $I$ for a diagram $\left(\square_{\cap}\right)$. We assume that all rings are finite-dimensional.

Theorem 1. Let $n \geq 1$ be an integer. For a diagram $\left(\square_{\cap}\right)$, consider the following statements:

(1) $R \subset T$ is 1-algebraic modulo $I$.

(2) $\operatorname{tr} . \operatorname{deg}[T / M: R /(M \cap R)]=0$ for each maximal ideal $M$ of $T$ containing $I$.

(3) $R \subset T$ is a residually algebraic extension.

(4) $R \subset T$ is universally algebraic modulo $I$.

(5) $R \subset T$ is n-algebraic modulo $I$.

(6) $R \subset T$ is 0 -algebraic modulo $I$.

Then:

(a) In general, (1) $\Leftrightarrow$ (2) $\Leftrightarrow$ (3) $\Leftrightarrow$ (4) $\Leftrightarrow$ (5) $\Rightarrow$ (6).

(b) If, in addition, $I \in \operatorname{Max}(T)$, then the above statements (1) - (6) are equivalent.

To prove the implications $(5) \Rightarrow(1)$ and $(5) \Rightarrow(6)$ in Theorem 1 , we need the following lemma.

Lemma 1. Let $n \geq 1$ be an integer. For a diagram ( $\square$ ), if $R \subset T$ is $n$-algebraic modulo $I$, then $R \subset T$ is $(n-1)$-algebraic modulo $I$.

Proof. Let $Q^{\prime} \subset Q$ be two prime ideals of $T[n-1]$ such that $I[n-1] \nsubseteq Q^{\prime}$ and $I[n-1] \subseteq Q$. Set $P^{\prime}=Q^{\prime} \cap R[n-1], P=Q \cap R[n-1]$ and suppose that $P^{\prime} \subset P$ are consecutive. Our task is to show that $R[n-1] / P \subseteq T[n-1] / Q$ is an algebraic extension. Let $\mathcal{Q}^{\prime}=Q^{\prime}+X_{n} T[n-1]\left[X_{n}\right]$ and $\mathcal{Q}=Q+X_{n} T[n-1]\left[X_{n}\right]$. It is obvious that $\mathcal{Q}^{\prime}$ respectively $\mathcal{Q}$ are uppers to $Q^{\prime}$ respectively $Q$. Set $\mathcal{P}^{\prime}=\mathcal{Q}^{\prime} \cap$ $R[n]$ and $\mathcal{P}=\mathscr{Q} \cap R[n]$. One can check easily that $\mathcal{P}^{\prime}=P^{\prime}+X_{n} R[n]$ and $\mathcal{P}=$ $P+X_{n} R[n]$. As $X_{n} R[n] \subseteq \mathcal{P}^{\prime} \subset \mathcal{P}$, then $\mathcal{P}^{\prime} \subset \mathcal{P}$ are consecutive. On the other hand, since $R \subset T$ is $n$-algebraic modulo $I$, then $\operatorname{tr} \operatorname{deg}[T[n] / \mathcal{Q}: R[n] / \mathcal{P}]=0$. As $T[n] / \mathcal{Q} \cong T[n-1] / Q$ and $R[n] / \mathcal{P} \cong R[n-1] / P$, it follows that $\operatorname{tr} . \operatorname{deg}[T[n-$ $1] / Q: R[n-1] / P]=0$, as desired.

Before proceeding to the proof of Theorem 1 it is convenient to recall the following Cahen's lemma [4, Proposition 4]. We shall make use of this result in the proof of 
Theorem 1. Note that this lemma holds even for polynomial rings since if $R:=$ $(T, I, D)$, then $R[n]:=(T[n], I[n], D[n])$.

Lemma 2. For a diagram ( $\square$ ), if $P_{0} \subset \ldots \subset P_{n}$ is a chain of primes in $R$ such that $P_{n}$ is minimal among primes of $R$ containing $I$ and $P_{n-1}$, then this chain lifts in $T$.

We now prove Theorem 1.

Proof of Theorem 1. (a) (1) $\Rightarrow$ (2) Let $\Omega$ be the finite subset of Max(T) such that $I=\bigcap_{M \in \Omega} M$. We discuss the following two cases.

Case 1. $|\Omega| \geq 2$. Since $M+\bigcap_{M^{\prime} \in \Omega \backslash\{M\}} M^{\prime}=T$, then there exist $u \in \bigcap_{M^{\prime} \in \Omega \backslash\{M\}} M^{\prime}$ and $v \in M$ such that $u+v=1$. Let $P_{1}^{\prime}=((X-u) T[X]) \cap$ $R[X]$ and $P_{1}=(M[X]+(X-u) T[X]) \cap R[X]$. The prime ideals $P_{1}^{\prime} \subset P_{1}$ are not necessarily consecutive. Since $T[X]$ is finite-dimensional, there exist two prime ideals $P^{\prime}$ and $P$ of $T[X]$ such that $P^{\prime}$ is maximal among the primes such that $P_{1}^{\prime} \subseteq P^{\prime} \subset P_{1}$ and not containing $I$, and $P$ is minimal such that $P_{1}^{\prime} \subseteq P^{\prime} \subset P \subseteq P_{1}$. Therefore $P^{\prime}$ does not contain $I, P$ contains $I$ and $P^{\prime} \subset P$ are consecutive. The chain $P_{1}^{\prime} \subseteq P^{\prime} \subset P$ lifts in $T[X]$ as $Q_{1}^{\prime} \subseteq Q^{\prime} \subset Q$. Notice that $Q_{1}^{\prime}=(X-u) T[X]$ because $P_{1}^{\prime}$ does not contain $I$ and so it lifts uniquely in $T[X]$. Hence $Q$ contains $X-u$ and $I$. The prime ideal $Q$ cannot contain any prime containing $u$ (if so, it would contain $X$, thus $X \in P_{1}$ and hence $u \in M$, which is absurd). Consequently $Q$ is above $M$. Furthermore $Q$ is an upper to $M$ because $X-u \in Q \backslash M[X]$. The prime ideal $P$ is above $p=M \cap R$. Next, we demonstrate that $P$ is an upper to $p$. Consider the polynomial $f=(X-u)(X-v)=X^{2}-X+u v$. Since $u v \in I$, then clearly $f$ belongs to $P_{1}^{\prime}=((X-u) T[X]) \cap R[X]$. Thus $f \in P$. As $f \notin p[X]$, we deduce that $P$ is an upper to $p$. As $R \subset T$ is 1 -algebraic modulo $I$, it follows that $T[X] / Q$ is algebraic over $R[X] / P$. Since $Q$ and $P$ are uppers respectively to $M$ and $p$, we deduce that $T / M$ is algebraic over $R / p$.

Case 2. $|\Omega|=1$. In this case $I=M$, where $M$ is a maximal ideal of $T$. The proof in this case proceeds along the same lines as in the proof of Case 1 with some modifications. Set $P_{1}^{\prime}=((X-1) T[X]) \cap R[X]$ and $P_{1}=(M[X]+(X-1) T[X]) \cap R[X]$. These prime ideals are not necessarily consecutive, so let $P^{\prime}$ be maximal among the primes such that $P_{1}^{\prime} \subseteq P^{\prime} \subset P_{1}$ and not containing $I$, and $P$ be minimal such that $P_{1}^{\prime} \subseteq P^{\prime} \subset P \subseteq P_{1}$. Therefore $P^{\prime}$ does not contain $I, P$ contains $I, P^{\prime} \subset P$ are consecutive and the chain $P_{1}^{\prime} \subseteq P^{\prime} \subset P$ lifts in $T[X]$ as $Q_{1}^{\prime}=(X-1) T[X] \subseteq Q^{\prime} \subset Q$. It is clear that $Q \cap T$ contains $I$, and as $I$ is a maximal ideal of $T$, then $Q \cap T=M$. Moreover, since $Q$ contains $X-1$, then $Q$ is an upper to $M$. The prime ideal $P$ is above $p=M \cap R$. We claim that $P$ is an upper to $p$. Consider the polynomial $f=(X-1)^{2}=X^{2}-2 X+1$. It is obvious that $f \in P_{1}^{\prime}=((X-1) T[X]) \cap R[X]$ and $f \notin p[X]$. Hence $f \in P \backslash p[X]$. Therefore $P$ is an upper to $p$ as claimed. Since $R \subset T$ is 1-algebraic modulo $I$, it results that $T[X] / Q$ is algebraic over $R[X] / P$. As $Q$ and $P$ are uppers respectively to $M$ and $p$, it follows that $T / M$ is algebraic 
over $R / p$.

(2) $\Rightarrow$ (3) Let $q \in \operatorname{Spec}(T)$. Our purpose is to show that $R /(q \cap R) \subseteq T / q$ is an algebraic extension. If $I \nsubseteq q$, then $T_{q} \simeq R_{q \cap R}$ (see [4, Proposition 0]). So $t r . d e g[T / q$ : $R /(q \cap R)]=0$. If $I \subseteq q$, then $q \in \Omega$. Hence $\operatorname{tr} \cdot \operatorname{deg}[T / q: R /(q \cap R)]=0$.

$(3) \Rightarrow(4) \Rightarrow(5)$ are trivial.

(5) $\Rightarrow$ (1) The conclusion is clear if $n=1$. So assume that $n \geq 2$. The conclusion follows readily from Lemma 1.

(5) $\Rightarrow$ (6) Follows readily from Lemma 1.

(b) We now assume that $I \in \operatorname{Max}(T)$. We will prove that $(6) \Rightarrow(2)$. To this end, we have only to show that $\operatorname{tr} \cdot \operatorname{deg}[T / I: R / I]=0$. Let $q^{\prime}$ be a prime ideal of $T$ such that $q^{\prime} \subset I$ are consecutive in $T$ (such ideal exists since $T$ is finite-dimensional). Let $p^{\prime}=q^{\prime} \cap R$, then $p^{\prime} \subset I$ are also consecutive in $R$. Indeed, assume that there exists a prime ideal $p$ of $R$ such that $p^{\prime} \subset p \subset I$. This chain lifts in $T$ to $q^{\prime} \subset q \subset I$ (notice that the unique prime ideal of $T$ lying over $I$ is $I$ itself since $I \in \operatorname{Max}(T))$. The desired contradiction since $q^{\prime} \subset I$ are consecutive. As $R \subset T$ is 0 -algebraic modulo $I$, then $\operatorname{tr} . \operatorname{deg}[T / I: R / I]=0$, as asserted.

Remark 1. If we leave out the assumption " $I \in \operatorname{Max}(T)$ " in the statement of Theorem 1 (b), the conclusion does not hold. More precisely, Fontana et al (see [8, Exemple 1.8]) have already constructed a diagram $(\square \cap)$, where $I$ is an intersection of two maximal ideals of $T$, such that $R \subset T$ is 0 -algebraic modulo $I$, whereas $R \subset T$ is not 1-algebraic modulo $I$.

\section{ACKNOWLEDGEMENT}

The authors would like to thank the referee for many valuable suggestions.

\section{REFERENCES}

[1] A. Ayache, "About a conjecture on Nagata rings," J. Pure. Appl. Algebra, vol. 98, pp. 1-5, 1995.

[2] A. Ayache and N. Jarboui, "On questions related to stably strong $S$-domains," J. Algebra, vol. 291, no. 3, pp. 164-170, 2005.

[3] M. Ben Nasr and N. Jarboui, "A counterexample for a conjecture about the catenarity of polynomial rings," J. Algebra, vol. 248, pp. 785-789, 2002.

[4] P.-J. Cahen, "Couple d'anneaux partageant un idéal," Arch. Math., vol. 51, pp. 505-514, 1988.

[5] P.-J. Cahen, "Construction $B, I, D$ et anneaux localement ou résiduellement de Jaffard," Arch. Math., vol. 54, pp. 125-141, 1990.

[6] M. Fontana, "Topologically defined classes of commutative rings," Ann. Mat. Pura Appl., vol. 123, no. 4, pp. 331-355, 1980.

[7] Fontana, M., Izelgue, L. and Kabbaj, S., "Quelques propriétés des chaines d'idéaux dans les anneaux $A+X B[X]$, , Commun. Algebra, vol. 22, no. 1, pp. 9-27, 1994.

[8] Fontana, M., Izelgue, L. and Kabbaj, S., "Sur quelques propriétés des sous-anneaux de la forme $D+I$ d'un anneau intègre," Commun. Algebra, vol. 23, no. 11, pp. 4189-4210, 1995.

[9] R. Gilmer, Multiplicative ideal theory, ser. Pure and Applied Mathematics. New York: Marcel Dekker, Inc., 1972, vol. 12.

[10] I. Kaplansky, Commutative Rings. Chicago: University Press, 1974. 
[11] I. Yengui, "Two counterexamples about the Nagata and Serre conjecture rings," J. Pure Appl. Algebra, vol. 153, no. 2, pp. 191-195, 2000.

[12] I. Yengui, "On questions related to saturated chains of primes in polynomial rings," J. Pure Appl. Algebra, vol. 178, no. 2, pp. 215-224, 2003.

Authors' addresses

Basma Gasmi

University of Sfax, Faculty of Sciences of Sfax, Department of Mathematics, P.O.Box: 1171, 3000 Sfax, Tunisia

E-mail address: basmagasmi@gmail.com

Noômen Jarboui

University of Sfax, Faculty of Sciences of Sfax, Department of Mathematics, P.O.Box: 1171, 3000 Sfax, Tunisia

E-mail address: noomenjarboui@yahoo.fr 